International Journal of Modern Physics E

(c) World Scientific Publishing Company

\title{
GAUSS-BONNET CONTRIBUTIONS TO THE ENTROPY OF EXTREMAL BLACK HOLES IN THE GAUGE-GRAVITY SECTOR
}

\author{
A. ULACIA REY. \\ Department of Theoretical Physics, ICIMAF, Calle 15 No-309 esq-E, Vedado. \\ La Habana, Plaza cp-10400, Cuba. \\ alain@icmf.inf.cu
}

Received (received date)

Revised (revised date)

\begin{abstract}
Using the Sen's mechanism we calculate the entropy for an $A d S_{2} \times S^{d-2}$ extremal and static black hole in four dimensions, with higher derivative terms that comes from a three parameter non-minimal Einstein-Maxwell theory. The explicit results for Gauss-Bonnet in the gauge-gravity sector are shown.
\end{abstract}

\section{Introduction}

There are several forms of introduce higher derivative terms taking into account the gauge-gravity sector. The standard way of perform the gauge-gravity interaction can be done in a minimal or non-minimal coupled picture. However, the gauge sector can be added in a linear or non-linear form. Finally, the gravitational sector can be added through a second order gravity or by terms of higher order of gravity. The more easy gauge-gravity theory is the Einstein-Maxwell 11 which is non-minimally coupled, is linear in the gauge sector and is of second order in derivative in the gravity sector. In this case we can find solutions like are the Reissner-Nordstrom (RN) black hole (BH) solution, and different models with electromagnetic fields 2 .

There are also other interesting solutions for minimally coupled gauge-gravity theory with non-linear gauge sector, and second order in gravity. They are the well known Born-Infeld models 3 , the Heisenberg-Euler 4 and more recently the Ayon-Beato and Garcia 5 solution.

The problem of the phenomenological introduction of non-minimal terms in the electrodynamic equations has been studied by Hehl and Obukhov 6 . Some modifications of Maxwell equations has been done by Drummond and Hathrell 7 from the one-loop corrections of quantum electrodynamics (QED) in curved spacetime.

In the following we will restrict our analysis to a coupling between gravity and electromagnetism in four dimensions with the restriction of work with second order field equations. This kind of coupling is called the Gauss-Bonnet (GB) coupling (or GB solution) in a gauge-gravity sector and it allows only one (independent) 
non-minimal coupling term in the action $[$. These modifications of the EinsteinMaxwell theory arises from higher dimensional Kaluza-Klein and string theory. In special, from a gravitational theory in five dimensions, via Kaluza-Klein dimensional reduction, separating the quartic terms in the Maxwell field 9 . The dimensional reduction of the Dirac action minimally coupled to gravity in five dimension leads a non-minimal coupling between Dirac field and the electromagnetic field 10 .

In other context, the entropy formalism ${ }^{11 \mid 12}$ has gained great importance because it has opened a new road for find the entropy of extremal BHs with $A d S_{2} \times S^{2}$ topology. In the last years the Sen mechanism 13 has been applied in several cases with nice results $14|15| 16$. For typical BPS BHs theses results for the entropy coincide with the statistical equation, the logarithm of the number of BPS micro-states. Such coincidences is one of the most important contributions of the string theory in the last years.

In the article 17 we calculated the entropy of extremal BHs using this mechanism and taking into account the complete set of Riemann invariants, with a minimal coupling between gravity and gauge. Thus when we work with invariants of higher order that comes from tensors as $R_{a b}, R_{a b c d}, C_{a b c d}, S_{a}$ a then we can say that working on the sector of pure gravity, although the gauge group $U(1)$ could be present. Therefore if we introduced gauge invariants of higher order like can be $\left(F^{a b} F_{a b}\right)^{2}, F^{a}{ }_{b} F^{b}{ }_{c} F^{c}{ }_{k} F^{k}{ }_{a}, \ldots$ we are referring to the pure gauge sector, then the gauge-gravity sector must be any combination of invariants, like could be $R F^{2}, g_{a b} F^{a b}, \ldots$ For simplicity, other invariants of covariant derivation like $R_{a}{ }^{b}{ }_{; c} R_{b}{ }^{a ; c}, S_{a}{ }^{b}{ }_{; c} S_{b}{ }^{a ; c}, \ldots$ or another will not be considered. This article constitutes our first step in introducing invariants from the gauge-gravity sector and obtain exact solutions of this. All calculations were carried out with the GR-Tensor package running on the algebraic Maple program.

This paper is organized in 4 Sections. First in Section 2 we consider the nonminimal coupled theory. Then in Section 3 we apply the Sen mechanism on the GB theory. Finally in Section 4 a brief conclusion is displayed.

\section{Non-minimal coupling theory with linear coupling in curvature}

A special case of non-minimal coupling is the theory with a restricts Lagrangian. It has a coupling between the electromagnetism and the metric which is linear in the curvature. Such action takes the form 18119 ,

$$
S=\int d x^{4} \sqrt{-g}\left(\frac{R}{16 \pi G_{4}}-\frac{F^{2}}{4}+\frac{w}{2} \chi^{i k m n} F_{i k} F_{m n}\right)
$$

\footnotetext{
aThey are respectively, the Ricci tensor, the Riemann tensor, the Weyl tensor, the Trace-free tensor and more ahead the Maxwell tensor $F_{i k}$.
} 
where $\chi^{i k m n}$, is the susceptibility tensol $\mathrm{b}$. The physics reason for the last term is that the induction tensor $H^{i k}$, and the Maxwell tensor $F_{m n}$ are linked by the linear law,

$$
H^{i k}=F^{i k}+\chi^{i k m n} F_{m n}
$$

as the magnetization tensor is defined by,

$$
4 \pi M^{i k}=H^{i k}-F^{i k} \equiv \chi^{i k m n} F_{m n}
$$

In the standard electrodynamics 20 the coefficients $\chi^{i k m n}$ form the non-minimal susceptibility tensor. The susceptibility tensor has the form,

$\chi^{i k m n}=\frac{q_{1} R}{2}\left(g^{i m} g^{k n}-g^{i n} g^{k m}\right)+\frac{q_{2}}{2}\left(R^{i m} g^{k n}-R^{i n} g^{k m}+R^{k n} g^{i m}-R^{k m} g^{i n}\right)+q_{3} R^{i k m n}$.

the parameter $q_{1}, q_{2}$ and $q_{3}$, are in general arbitrary. They can be chosen phenomenologically or by other way. At the same time, they introduce cross-terms which describe non-minimal interactions of the electrodynamics and gravitational fields. For example, the Drummond-Hathrell ${ }^{7}$ constraint modified the Maxwell equations from one-loop correction of QED in curved space-time, in this case the coupling constants can be written as, $w=1,2 q_{1}-q_{3}=0,13 q_{1}+q_{2}=0, q_{1}=$ $-\alpha \lambda_{e}^{2} / 180 \pi$, where $\alpha$ is the fine structure constant and $\lambda_{e}$ is the Compton wavelength of the electron. If $q_{1}=q_{2}=q_{3}=w=0$, in Eq. (4) the RN solution is displayed.

The Gauss-Bonnet model 2122 (in the context of gauge-gravity sector) is obtained when is request the proportionality between the susceptibility tensor and the double-dual Riemann tensor $\chi_{i k m n}=q R_{i k m n}^{* *}$. Here the Einstein-Maxwell coupling equations are second order in the derivatives, for that reason is called Gauss-Bonnet model, it is also an one-parameter model with $q_{1}=q_{3}=-1, q_{2}=2$ and $w=q$.

In general these cross-terms in the Lagrangian represent the interactions between gravity and electromagnetic forces at non-minimal level, therefore they means that the velocity of the gauge-gravity waves differ from the speed of the light in the vacuum.

\section{Applying the Sen mechanism}

The Sen mechanism has allowed to compute the entropy for extremal BH in theories with higher derivative terms. There are several examples that prove the efficiency of this mechanism.

The more general metric for an extremal and stationary $\mathrm{BH}$ with $A d S_{2} \times S^{2}$ topology in 4-dimensions is,

${ }^{\mathrm{b}}$ All the other quantities are commonly well-known, $R$ is the Ricci scalar, $G_{4}$ the Newton constant and $w$ the gauge-gravity coupling constant. 


$$
\begin{aligned}
& d s^{2}=v_{1}\left(-r^{2} d t^{2}+\frac{d r^{2}}{r^{2}}\right)+v_{2}\left(d \theta^{2}+\sin ^{2} \theta d \phi^{2}\right), \\
& F_{r t}=e, \quad F_{\theta \phi}=p \sin \theta / 4 \pi .
\end{aligned}
$$

where $v_{1}, v_{2}$ are related with the geometry of the $\mathrm{BH}$ throat, $e$ and $p$ are related with the $\mathrm{BH}$ charges. Is important to point out that we are assuming our gauge-gravity theory in connexion with the $A d S_{2} \times S^{2}$. This means that our theory must contain the RN solutiond that at the extreme limit of larger charges, near the horizon of geometry could be connected with the $A d S_{2} \times S^{2}$ topology and the associated isometry $S O(2,1) \times S O(3)$. Furthermore, is natural postulate that the effects of added higher derivative terms from the gauge-gravity sector will not destroy the symmetries near the horizon of geometry 1323 . Thus let us define $\mathcal{E}$ (the entropy function) like,

$$
\mathcal{E}(\vec{v}, \vec{e}, \vec{q}, \vec{p})=2 \pi\left(e_{i} q_{i}-f(\vec{v}, \vec{p}, \vec{e})\right)
$$

where $f$ is the Lagrangian density evaluated for the near horizon of geometry. $\mathcal{E}$ contains all the information near the horizon of the geometry. Thus all the extremal conditions of the parameter determine the equations of motion and the conservation near the horizon of geometry. The value of $\mathcal{E}$ on the horizon of geometry, is the entropy of the $\mathrm{BH}$.

If we calculate these quantities for a gauge-gravity non-minimally coupled theory in the Gauss-Bonnet case we get for the entropy function,

$$
\mathcal{E}=-\left(\frac{16 \pi^{2} e^{2}}{v_{1}}+\frac{p^{2}}{v_{2}}\right) w+2 \pi e q-\frac{4 \pi^{2} e^{2} v_{2}}{v_{1}}+\frac{v_{1} p^{2}}{4 v_{2}}-\frac{\pi\left(v_{1}-v_{2}\right)}{G_{4}}
$$

The equations of motion are determined by extremizing the entropy function,

$$
\frac{\partial \mathcal{E}}{\partial v_{j}}=\frac{\partial \mathcal{E}}{\partial e}=0, \quad \text { with } \quad j=1,2 .
$$

Therefore the equations of motion near the horizon of geometry can be written as follows,

$$
\begin{aligned}
\frac{-16 \pi^{2} e^{2} w}{v_{1}^{2}}-\frac{p^{2}}{4 v_{2}}+\frac{\pi}{G_{4}}-\frac{4 v_{2} e^{2} \pi^{2}}{v_{1}^{2}} & =0, \\
\frac{p^{2} w}{v_{1}^{2}}-\frac{v_{1} p^{2}}{4 v_{2}^{2}}+\frac{\pi}{G_{4}}-\frac{4 \pi^{2} e^{2}}{v_{1}} & =0, \\
\frac{-e w}{v_{1}}+\frac{q}{16 \pi}-\frac{v_{2} e}{4 v_{1}} & =0 .
\end{aligned}
$$

Thus when we solve this system of equations we get two set of solutions. One of them can be discarded because it does not contain the RN solution, hence we get

${ }^{\mathrm{c}}$ If $w=0$ in Eq. 1 the RN solution is obtained. 
of extremal conditions for,

$$
\begin{aligned}
& v_{1}=v_{2}+4 w, \quad e=\frac{q}{4 \pi} \\
& v_{2}=\frac{\left(G_{4}\left(p^{2}+q^{2}\right)-16 \pi w\right)}{8 \pi}\left[1+\sqrt{1+\frac{4^{3} \pi w G_{4} p^{2}}{16 \pi w-G_{4}\left(p^{2}+q^{2}\right)}}\right] .
\end{aligned}
$$

Substituting this into the expression for $\mathcal{E}$ in (8) we get,

$$
S_{G B}=\frac{\pi v_{2}}{G_{4}}-\frac{p^{2} w}{v_{2}}
$$

Clearly, the Eqs.(13)-(14) reproduces the RN solution when $w=0$. But from Eq. (13), we can see that the introduction of the higher derivative terms in the action makes a tiny change in the geometry of the BH throat. Normally, under this limit, near the horizon of geometry, the coupling constant must be inverse to the powers of the electric and magnetic charges. Therefore, the correction to the entropy of $\mathrm{RN}$ is small in Eq. (14). This GB solution for entropy is contained in the gaugegravity sector and introduce the same effect than the typical GB in the sector of pure gravity. Both modify the entropy of Bekenstein-Hawking. Note that the GB solution in the sector of pure gravity takes form 2417 ,

$$
\begin{aligned}
v_{1} & =v_{2}, \quad e=\frac{q}{4 \pi}, \quad v_{2}=\frac{G_{4}}{4 \pi}\left(p^{2}+q^{2}\right), \\
S_{G B}^{g r a v} & =\frac{\pi v_{2}}{G_{4}}+64 \pi^{2} \alpha .
\end{aligned}
$$

where $\alpha$ is the coupling constant. This solution does not change the throat topology

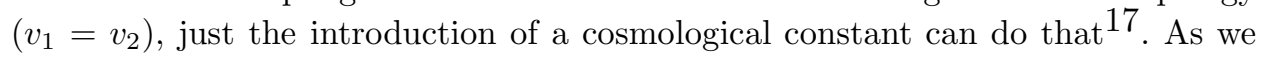
supposed, near the horizon of geometry the coupling constant is inverse to the $\mathrm{BH}$ charge, then its contribution in Eq (16) is also small.

\section{Conclusions}

The Sen mechanism let us to find exact results for the entropy of extremal BH in four dimensions with $A d S_{2} \times S^{2}$ topology, taking into account terms of higher derivatives in the sector of gauge-gravity of a GB theory. These results were compared with the similar ones in the sector of pure gravity of the GB theory. In both cases and with small contributions, the area law is modified.

\section{Acknowledgements}

The author acknowledge thanks the Office of External Activities of ICTP for its support through NET-35. Also thank to CLAF (Latin-American Center for Physics) and Cuban Physics Society for their contributions and supports in the organization of the events STARS-2011 and SMFNS-2011. 
A. ULACIA REY

\section{References}

1. Misner C W, Thorne K S and Wheeler J A Gravitation (W H Freeman and Company, NY, 1973).

2. Wald R M General Relativity (University of Chicago Press Ltd, 1984).

3. Born M and Infeld L Proc. Roy. Soc. (London) A 144 (1934) 425.

4. Heisenberg W and Euler H Z. Physik 98 (1936) 714.

5. Ayón-Beato E and García A Phys. Rev. Lett 80 (1998) 5056.

6. Hehl F W and Obukhov Yu N Lect. Notes Phys. 562 (2001) 479.

7. Drummond I T and Hathrell S J Phys. Rev. D 22 (1980) 343.

8. Horndeski G J. Math. Phys. 17 (1976), 1980.

9. Buchdahl H J. Phys. A: Math. Gen. 12 (1979) 1037. Müller-Hoissen F Phys.Lett. 201B (1988) 325.

10. Pauli W Ann. Phys. Lpz. 18 (1933) 337. Souriau J-M Nuovo Cimento 30 (1963) 565.

11. Ferrara S and Kallosh R Phys. Rev. D 54 (1996) 1514.

12. Ferrara S, Kallosh R and Strominger Phys. Rev. D 52 (1995) 5412.

13. Sen A Gen. Rel. Grav. 40 (2008) 2249.

14. Sen A JHEP 0509 (2005) 038; Sen A JHEP 0603 (2006) 008.

15. Ghodsi A Phys. Rev. D $\mathbf{7 4}$ (2006) 124026.

16. Prester P JHEP 0602 (2006) 039; Sinha A and Suryanarayana N V Class.Quant. Grav. 23 (2006) 3305; Sahoo B and Sen A JHEP 0609 (2006) 029; Exirifard G JHEP 0610 (2006) 070; Chandrasekhar B arXiv:hep-th/0604028. Cai R G and Pang D W Phys. Rev. D 74 (2006) 064031; Sinha A and Suryanarayana N V JHEP 0610 (2006) 034; Sahoo B and Sen A JHEP 0701 (2007) 010; Cardoso G L, Oberreuter J M and Perz J arXiv:hep-th0701176; Goldstein K and Jena R P arXiv:hep-th0701221 ; Cai R G and Pang D W arXiv:hep-th0702040; Alishahiha M arXiv:hep-th0703099; Cai R G, Chen C M, Maeda K I, Ohta N and Pang D W Phys.Rev.D 77 (2008) 064030.

17. Ulacia Rey A JHEP 08 (2009) 107.

18. Balakin Alexander B Class. Quant. Grav. 22 (2005) 1867-1880.

19. Balakin Alexander B Phys.Rev.D 77 (2008) 084013.

20. Landau L D, Lifchitz E M and Pitaevskii L P Electrodynamics of Continuous Media (Butterworth Heinemann, Oxford, 1996).

21. Horndeski G Phy. Rev. D 17, (1978) 391.

22. Müller-Hoissen F and Sippel R Class. Quantum. Grav. 5 (1988) 1473.

23. Kunduri H K, Lucietti J and Reall H S, Class. Quant. Grav 24 (2007) 4169-4190.

24. Morales J F and Samtleben H JHEP 0610 (2006) 074. 